www.unibe.ac.cr/rfp

\title{
Intensidad de uso de Facebook y habilidades sociales en adolescentes
}

\author{
Mariana Agüero \\ Universidad de Iberoamerica
}

\section{Resumen}

El uso cada vez mayor de las redes sociales electrónicas ha sido objeto de distintos estudios y es importante recalcar como muchas de esas investigaciones se relacionan a los efectos adversos que pueden tener las personas que diariamente permanecen en Facebook por muchas horas al día. (Las Heras, 2012). El presente artículo consiste en una investigación sobre la frecuencia del uso de la red social Facebook en relación con las habilidades sociales de los y las adolescentes del Ministerio Jóvenes en Victoria. Se utilizó una metodología cuantitativa no experimental-transversal, la población a estudiar fueron adolescentes en edades entre los 13 y 18 años $(\mathrm{M}=15, \mathrm{DE}=1.6)$, del grupo Jóvenes en Victoria. Para la recolección de información se utilizó el Cuestionario de Evaluación de Dificultades Interpersonales en la Adolescencia (CEDIA) (Inglés, Méndez y Dolores, 2000) y una encuesta de Frecuencia de uso de Facebook elaborada creada para el presente estudio. Dentro de los resultados se destacaron importantes correlaciones con respecto a las variables planteadas en la investigación, se determina que no existe una relación directa entre las habilidades sociales percibidas y las horas invertidas en la red social Facebook.

Palabras Clave: Redes sociales, habilidades sociales, adolescentes

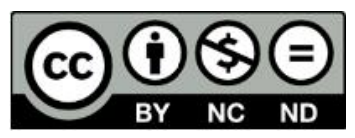


Agüero

\section{Introducción}

Hoy en día, personas de todas las edades y en especial los y las jóvenes tienen la necesidad de utilizar las redes sociales con diversos fines, como compartir con sus amigos, conocer gente nueva, compartir sus intereses, entre otros. El uso acentuado de Internet en el establecimiento de relaciones a partir de las redes sociales, ha aportado un cambio significativo al tipo de encuentro que puede generar entre las personas ya que permite las relaciones entre las mismas con el objetivo de estar en contacto con personas conocidas y desconocidas, apoyo emocional, compañía social. Además les permite por medio de una identidad digital, construir, intercambiar intereses y socializar (Las Heras, 2012).

Las redes sociales en la Internet han generado un cambio importante en cómo las personas se comunican entre ellas mismas, ahora es mucho más fácil estar al tanto de las demás personas con respecto a su vida personal y en cualquier parte del mundo. Como producto de la globalización, el uso de las mismas es de suma importancia hoy en día, ya que se le han dado múltiples usos como por ejemplo muchas empresas se dan a conocer por Facebook dándosele a las personas un trato más personal, igual los artistas, programas de televisión, entre otras, en fin, Facebook se ha convertido en una herramienta indispensable para la vida cotidiana de las personas, ha influido en nuestro vocabulario, en nuestra forma de comunicarnos, pero sobretodo en la forma en la que nos relacionamos socialmente ya que hemos transformado la forma de hacer amistades, hemos cambiado la manera de publicar nuestras relaciones, la forma de reunir a grupos de gente, y de compartir información personal con nuestros contactos (Las Heras, 2012). 
Al observar el impacto social y comunicacional que están teniendo las redes sociales electrónicas, es de suma importancia que se realicen investigaciones que busquen entender y explicar los efectos en la dinámica y formas de comunicarse que hacen un cambio considerable en lo denominado habilidades sociales, además de cómo difieren las relaciones interpersonales en línea comparada con la relación cara a cara.

La empresa UNIMER (2010), realizó un estudio en Costa Rica sobre el uso de las redes sociales. Realizaron una encuesta a 1210 personas costarricenses con edades entre los 18 a 69 años en todo el territorio nacional. Dentro de los resultados se mencionan que $(18,9 \%)$ declaró que pertenece a alguna red social, siendo Facebook la favorita con (76\%) de los participantes. Con respecto a la frecuencia del uso un (17\%) de los usuarios de redes sociales ingresan varias veces al día, mientras que otro (21\%) ingresa una vez al día y los momentos más frecuentes para acceder a redes sociales son la noche (45\%) o la tarde (35\%). Además (38\%) de los usuarios declararon que usan la red social durante una hora al día, mientras que un $(31 \%)$ respondió que el período es inferior a 30 minutos. Entre las personas con trabajo remunerado el tiempo promedio es de 72 minutos. Unos 349 mil chatean con amigos, mientras que unos 36 mil siguen empresas. Otro uso importante realizado por unos $218.6 \mathrm{mil}$ adultos es subir o bajar fotos, mientras que unos 116.5 mil personas indicaron que aprovechan las redes sociales para buscar amigos o ex compañeros o estar en contacto con ellos, el (81\%) de las personas utiliza las redes sociales como un medio para relacionarse con otras personas.

Por otro lado la empresa Socialbakers (2012), menciona que en Costa Rica un (95\%) de los usuarios web poseen una cuenta en por lo menos una red social, siendo Facebook la red social preferida $(92,1 \%)$. Actualmente hay 1935740 usuarios costarricenses registrados en Facebook, lo que significa una penetración del (42.86\%) de la población total del país y 
Agüero

de un $96,79 \%$ de la población usuaria de internet. Llama la atención que 158 mil perciben el beneficio de hablar sobre diferentes temas con libertad, mientras que 30 mil consideran que les permite hacer comentarios que no harían en persona. Las personas también experimentan control, respeto y un sentido de aceptación, emociones que se relacionan con las necesidades universales del ser humano, el poder y la pertenencia.

Un estudio realizado por las autoras Herrera et al. (2010), compara la adicción al Facebook con la depresión, autoestima y habilidades sociales en universitarios de México. Encontrando que los adictos a Facebook tienden a tener puntuaciones más altas, es decir, tienen mayor propensión a la depresión, no poseen muchas habilidades sociales y su autoestima es baja en comparación con los no adictos.

Por su parte Zywica y Danowski (2008), plantearon dos hipótesis en donde mencionan que el facebook ayuda al mejoramiento social ("El rico se vuelve más rico") en personas que son populares fuera de Facebook. Y la segunda hipótesis, la Compensación Social ("El pobre se vuelve más rico"), en este caso sería que Facebook ayuda a incrementar la popularidad compensando la no popularidad cuando esta fuera del mismo (Zywica y Danowski, 2008). Los resultados mencionan que los usuarios más extrovertidos tienden a ser populares en Facebook y fuera de este, mientras que las personas más introvertidas, lucharon por ser más populares en Facebook.

En Canadá se realizó un estudio para examinar la influencia de los factores de la personalidad y competencias en el uso de Facebook (Ross et al, 2009). Como resultados encontraron que la personalidad se asocia con algunos aspectos del uso de Facebook, por ejemplo los individuos con rasgos de extroversión pertenecen a muchos grupos sociales en Facebook, por lo que se concluyó que son más propensos a participar en actividades sociales, un dato interesante es que las personas que resultaron ser extrovertidas no se asociaron 
directamente con el numero de amigos que tengan, por lo que los autores mencionan que utilizan el Facebook como una herramienta social, no como una alternativa a las actividades sociales.

A raíz de lo anterior nace el problema de investigación que plantea lo siguiente:

¿Existe relación entre el tiempo invertido en el uso de la red social Facebook y las habilidades sociales de los y las adolescentes del Ministerio Jóvenes en Victoria? La hipótesis central consiste en que no existe relación entre el tiempo invertido en el uso de la red social Facebook y el nivel de las habilidades sociales de las y los adolescentes.

\section{Método}

\section{Participantes}

En el estudio participaron 100 jóvenes entre 13 a 18 años $(M=15, D E=1.6)$, del Ministerio Jóvenes en Victoria.

\section{Instrumentos}

Los instrumentos utilizados con el fin de poder analizar los resultados de los objetivos propuestos fueron el Cuestionario de Evaluación de Dificultades Interpersonales en la Adolescencia (CEDIA) (Inglés, Méndez y Dolores, 2000) y una escala para la medición del uso de Facebook construida para esta investigación.

Para cuantificar el uso de Facebook se elaboró una encuesta de 4 sub-escalas sobre el uso de Facebook en escala Likert con niveles de 1 a 5 y opción múltiple. Se obtuvo a nivel general una confiabilidad aceptable $(\alpha=.86)(\mathrm{M}=2.5, \mathrm{DS}=0.6)$; En cuanto la sub-escala 
Agüero

dedicada a medir los niveles de entretenimiento se obtuvo una confiabilidad aceptable $(\alpha=.70)(M=2.9, D S=0.7)$, por otro lado la sub escala Demostrar Afecto presenta una confiabilidad elevada de $\alpha=.89(\mathrm{M}=2.4, \mathrm{DS}=1.1)$.

La sub-escala Moda presenta una confiabilidad aceptable de $\alpha=.70(\mathrm{M}=2.2, \mathrm{DS}=1.1)$, Las sub-escalas Compartir problemas y Sociabilidad presentaron una consistencia de $\alpha=.72$ $(\mathrm{M}=2.3, \mathrm{DS}=1.1)$ y $\alpha=.73(\mathrm{M}=2.1, \mathrm{DS}=1.0)$, respectivamente. En cuando al factor Información social consta de un nivel de confiablidad elevado $(\alpha=.81)(\mathrm{M}=2.7, \mathrm{DS}=1.0)$.

Por último las escalas Motivo de uso y Frecuencias de uso presentan una confiabilidad optima $(\alpha=.73)(\mathrm{M}=2.8, \mathrm{DS}=0.7)$ y de $(\alpha=.76)(\mathrm{M}=2.7, \mathrm{DS}=0.7)$, respectivamente.

Las sub-escalas anteriormente expuestas constan de 10 reactivos, formulados por medio de escalas tipo Likert.

El Cuestionario de Evaluación de Dificultades Interpersonales en la Adolescencia (CEDIA) (Inglés, Méndez y Dolores, 2000), está compuesto por 39 ítems distribuidos en cinco factores: Aserción ( $\alpha=.74)$, Relaciones con el otro sexo $(\alpha=.70)$, Relaciones con Iguales $(\alpha=.78)$, Hablar en Público $(\alpha=.65)$ y Relaciones Familiares $(\alpha=.66)$. Estas dos últimas escalas se omitieron para propósitos de este estudio debido a la baja consistencia de las escalas y la irrelevancia con los objetivos de la investigación. Los ítems están establecidos en una escala tipo Likert de rango de 0 (ninguna dificultad) a 4 (máxima dificultad).

Se agregó una sección de datos sociodemográficos (género, edad, tipo de institución educativa) para obtener la distribución de la muestra

\section{Resultados}


Para determinar la relación entre la satisfacción percibida con las habilidades sociales en usuarios de alto y bajo uso de la red social Facebook, se realizó un análisis de correlación entre la escala de habilidades sociales (CEDIA) y la variable que mide las horas que el usuario invierte semanalmente y así determinar si se cumple la hipótesis (Ver Tabla 1).

Tabla 1: Medias, desviaciones típicas y correlaciones simples entre Habilidades Sociales y las demás variables de estudio

\begin{tabular}{lcccc}
\hline \multicolumn{1}{c}{ Variable } & $\alpha$ & $\mathrm{M}$ & $\mathrm{DE}$ & Habilidades Sociales \\
\cline { 2 - 5 } Horas invertidas en Facebook & --- & 39 & 36.7 & .17 \\
Tipos de uso & .86 & 2.5 & 0.6 & .14 \\
Frecuencia del uso de las aplicaciones & .76 & 2.8 & 0.7 & .11 \\
Motivos de uso & .73 & 2.8 & 0.7 & .14 \\
\hline
\end{tabular}

A partir de los resultados se determina que no existe una relación directa entre las habilidades sociales percibidas y las horas invertidas en la red social $(r=.18 ; p=.067)$, a su vez las habilidades sociales tampoco se relacionan con los tipos de uso de la red social $(r=14$; $p=.155)$, con la frecuencia invertida en los diferentes aplicaciones de Facebook $(r=.11$; $p=.245)$ y los motivos de uso $(r=14 ; p=.146)$.

A pesar de lo anterior las habilidades sociales se relacionan con las sub escalas moda $(\mathrm{r}=.20 ; \mathrm{p}=.03)$ y compartir problemas $(\mathrm{r}=-.23 ; \mathrm{p}=.01)$ de la escala de usos de Facebook (Ver tabla 2).

Tabla 2. Medias, desviaciones típicas y correlaciones simples entre Habilidades Sociales y la variable en estudio.

\begin{tabular}{llllc}
\hline Variable & $\alpha$ & M & DE & Habilidades Sociales \\
\cline { 2 - 5 } Moda & .70 & 2.2 & 1.1 & $.20^{*}$ \\
Compartir problemas & .72 & 2.3 & 1.1 & $.23^{*}$ \\
\hline
\end{tabular}


Agüero

$* \mathrm{p}<0.001$

Lo anterior indica que a menores habilidades sociales los jóvenes tenderán a utilizar Facebook por moda y para compartir problemas en comparación con los jóvenes que poseen buenas habilidades sociales.

Siguiendo con los objetivos de la investigación y su línea de análisis, las variables sexo $(\mathrm{r}=-.01 ; \mathrm{p}=.89)$ edad $(\mathrm{r}=.03 ; \mathrm{p}=.69)$ y año académico $(\mathrm{r}=.09 ; \mathrm{p}=.34)$ no muestran una relación directa con el grado de habilidades sociales que tienen los y las adolescentes del Ministerio Jóvenes en Victoria (Ver tabla 3).

Tabla 3: Medias, desviaciones típicas y correlaciones simples entre Habilidades Sociales y las variables en estudio

\begin{tabular}{lcccc}
\hline Variable & $\alpha$ & M & DE & Habilidades Sociales \\
\cline { 2 - 5 } Sexo & --- & 2 & .5 & -.01 \\
Edad & --- & 15 & 1.6 & .03 \\
Año Académico & --- & 3 & 1.3 & .09 \\
\hline
\end{tabular}

Aunado a lo anterior se realizó un análisis para determinar si existe una relación entre los motivos por los cuales se utiliza la red social Facebook, relacionado con la frecuencia en que los jóvenes utilizan las aplicaciones que contiene dicha red social (Ver tabla4).

Tabla 4: Medias, desviaciones típicas y correlaciones simples entre Motivos de uso y la variable en estudio.

Variable

Frecuencia del uso de las aplicaciones $\alpha \quad \mathrm{M}$ DE Motivos de Uso

\begin{tabular}{llll}
\hline 76 & 2.8 & 0.7 & $.71 *$
\end{tabular}




$$
* \mathrm{p}<0.001
$$

Dicho análisis muestra que existe una relación positiva entre los motivos para el uso de Facebook y la frecuencia del uso de las aplicaciones $(r=.71 ; p<.001)$. Lo anterior indica que a mayor grado de motivos para utilizar una red social, mayor va a ser la frecuencia con la que se ingresa y se utilizan las características que brinda la misma.

Con el propósito de conocer si la cantidad de horas que se conectan los jóvenes se relaciona con el agregar personas conocidas y desconocidas. Se realizó una correlación bivariada tomando en cuenta la variable "cantidad de horas que se conectan" y la variable "A quién añade a su lista de amigos" (Ver tabla 5).

Tabla 5: Medias, desviaciones típicas y correlaciones simples entre Horas de uso y las variables en estudio

\begin{tabular}{lcccc}
\hline Variable & $\alpha$ & $\mathrm{M}$ & $\mathrm{DE}$ & Horas de Uso \\
\cline { 2 - 6 } Añado a los amigos que conozco en la vida real & --- & 1.8 & 0.4 & -.15 \\
A los amigos de mis amigos & --- & 1.6 & 0.4 & $.29 *$ \\
A la gente que no conozco & --- & 1.3 & 0.4 & $.24^{*}$ \\
\hline p $<0.001$ & & & &
\end{tabular}

La anterior tabla determina que existe una relación positiva entre la variable de cantidad de horas semanales y añadir a los amigos de mis amigos $(r=.29 ; \mathrm{p}<0.003)$ y añadir a la gente que no conozco $(r=.24 ; \mathrm{p}<0.015)$, no así con añadir personas que conocen en la vida real. Demostrando así que a mayor tiempo invertido semanalmente, más personas desconocidas agregarán. 
Agüero

En referencia al último objetivo de la investigación, se procedió a realizar un análisis de correlación entre las horas invertidas semanalmente y la frecuencia del uso de las aplicaciones, los motivos del uso y los tipos de uso (Ver tabla 6).

Tabla 6: Medias, desviaciones típicas y correlaciones simples entre Horas de uso y las variables en estudio

\begin{tabular}{lcccc}
\hline \multicolumn{1}{c}{ Variable } & A & M & DE & Horas de Uso \\
\cline { 2 - 5 } Frecuencia del uso de las aplicaciones & .76 & 2.5 & 0.6 & $.34^{*}$ \\
Motivos del uso & .73 & 2.8 & 0.7 & $.25^{*}$ \\
Tipos del uso & .86 & 3 & 1.3 & $.28^{*}$ \\
\hline$* \mathrm{p}<0.001$ & & & &
\end{tabular}

Los resultados arrojan que existe una relación positiva entre la variable de horas semanales y la frecuencia de las aplicaciones $(\mathrm{r}=.34 ; \mathrm{p}<0.001)$, los motivos del uso $(\mathrm{r}=.25$; $\mathrm{p}=0.011)$ y los tipos de uso $(\mathrm{r}=.28 ; \mathrm{p}=0.004)$. Demostrando así que a mayor tiempo invertido semanalmente va a influir directa y positivamente en los tipos de uso, frecuencia y motivos. Para determinar los predictores de las habilidades sociales y de la frecuencia del uso de Facebook se realizó un análisis de regresión jerárquica con el método Stepwise (Ver Tabla 7 y 8). Con respecto a las habilidades sociales, el modelo general de estos predictores explica un $18 \%$ de la varianza, $\mathrm{F}(3,91)=6.75, \mathrm{p}<.001$. Las habilidades sociales fueron predichas principalmente por compartir problemas $(\beta=.26, \mathrm{p}=.009)$, luego de manera inversa por añadir amigos en la vida real $(\beta=-.20, \mathrm{p}=.038)$, por último, de forma inversa por cuantos amigos de los que añade conoce en la vida real $(\beta=-.19, \mathrm{p}=.039)$ (Ver tabla 7). 
Tabla 7: Resumen de regresión jerárquica para las variables que pronostican las habilidades sociales $(n=100)$.

\begin{tabular}{|c|c|c|}
\hline \multicolumn{3}{|c|}{ Modelo 1} \\
\hline $\mathrm{R}_{2}=.09, \mathrm{~F}=9.62, \mathrm{gl}=1$ & & \\
\hline$\beta$ & Compartir problemas ${ }^{* *}$ & .306 \\
\hline \multicolumn{3}{|c|}{ Modelo 2} \\
\hline \multicolumn{3}{|l|}{$\mathrm{R}_{2}=.14, \mathrm{~F}=7.659, \mathrm{gl}=2$} \\
\hline \multirow[t]{2}{*}{$\beta$} & Compartir problemas ${ }^{*}$ & .251 \\
\hline & Agregar amigos que conoce en la vida real ${ }^{*}$ &.-228 \\
\hline \multicolumn{3}{|c|}{ Modelo 3} \\
\hline \multicolumn{3}{|l|}{$\mathrm{R}_{2}=.18, \mathrm{~F}=6.753, \mathrm{gl}=3$} \\
\hline \multirow[t]{3}{*}{$\beta$} & Compartir problemas ${ }^{* *}$ & .260 \\
\hline & Agregar amigos que conoce en la vida real ${ }^{*}$ & -.207 \\
\hline & Cantidad de amigos que conoce en la vida real ${ }^{*}$ & -.199 \\
\hline
\end{tabular}

Lo anterior se interpreta afirmando que los jóvenes que entre más agreguen y tengan de amigos, personas que conozcan en la vida real y menos utilicen Facebook para compartir problemas más habilidades sociales tendrán los adolescentes del Ministerio Jóvenes en Victoria. En relaciona a las horas invertidas, el modelo general de estos predictores explica un $18 \%$ de la varianza, $F(2,91)=9,66, \mathrm{p}<.001$. Se comprobó que la cantidad de horas diarias se predice principalmente por la frecuencia invertida en las diferentes opciones de comunicación de la red social Facebook $(\beta=.28, \mathrm{p}=.006)$, también con respecto a los amigos de sus amigos que añade $(\beta=.25, \mathrm{p}=.012)($ Ver tabla 8$)$.

Tabla 8: Resumen de regresión jerárquica para las variables que pronostican las horas invertidas en facebook $(n=100)$.

\begin{tabular}{ll}
\hline $\mathrm{R}_{2}=.10, \mathrm{~F}=12.014, \mathrm{gl}=1$ & Modelo 1 \\
\end{tabular}


Agüero

\begin{tabular}{ccc}
$\beta$ & Frecuencia del uso de las aplicaciones $^{* *}$ & .340 \\
\hline $\mathrm{R}_{2}=.15, \mathrm{~F}=9.660, \mathrm{gl}=2$ & Modelo 2 & \\
$\beta$ & Frecuencia del uso de las aplicaciones & \\
& & .278 \\
${ }^{* *} p<0.001 ;{ }^{*} p<.12$ & Agregar amigos de sus amigos & .252 \\
\hline
\end{tabular}

Lo cual se interpreta afirmando que los jóvenes que utilizan con frecuencia Facebook, son más propensos a compartir y ver videos, subir fotografías, ver los perfiles de sus amigos, actualizar su perfil, jugar, utilizar aplicaciones, utilizar el chat, hacer comentarios y dar "likes" a los estados de las demás personas, unirse a páginas, cambiar el estado; además de agregar a personas que no conocen.

\section{Discusión}

El uso de Internet y de las redes sociales, como Facebook, es un tema que tiene que empezar a ser estudiado de manera meticulosa, ya que cada día saltan al escenario de la Web, incontables usuarios que tienen un tipo de cuenta dentro de ellas. Hoy en día, estos medios de comunicación son utilizados de manera frecuente por adolescentes, lo cual invariablemente cambia según la cultura y el país, y depende del acceso a Internet, el número de horas de tiempo libre y otros factores.

El uso cada vez mayor de las redes sociales electrónicas ha sido objeto de distintos estudios y es importante recalcar como muchas de esas investigaciones se relacionan a los efectos adversos que pueden tener las personas que diariamente permanecen en Facebook por muchas horas al día y entre esas consecuencias se encuentra las habilidades sociales pobres, por ejemplo, la empresa UNIMER (2010), menciona que 158 mil de las personas 
entrevistadas, perciben el beneficio de hablar sobre diferentes temas con libertad en Facebook, mientras que 30 mil consideran que les permite hacer comentarios que no harían en persona. Sin embargo, también, las personas experimentan control, respeto y un sentido de aceptación, emociones que se relacionan con las necesidades universales del ser humano, el poder y la pertenencia.

Además es interesante ver como en ciertos artículos mencionan el término de adicción a Facebook en personas que pasan más de 5 horas conectadas a las redes sociales durante el día y que a raíz de lo anterior las personas son más propensas a tener pobres habilidades sociales, depresión y baja autoestima (Herrera et al, 2010), también en otro estudio se reportan datos referentes a las relaciones interpersonales, en donde se menciona que: "los sujetos adictos mantienen relaciones poco cambiantes, les incomoda la soledad y pueden utilizar la conexión a la red como acompañamiento" (Jiménez y Pantoja, 2007), en cambio los sujetos que no lo son, pueden mantener relaciones estables y pueden tolerar la soledad, además de establecer relaciones amorosas más sanas, los mismos "muestran predominantemente motivaciones individuales a la conexión ya que sus necesidades sociales parecen satisfacerse por otras vías" (Jiménez y Pantoja, 2007).

Por lo que se puede interpretar de lo anterior que las habilidades sociales si van a ser influenciadas de manera positiva o negativa, por la cantidad de horas que los usuarios se conectan al día. Sin embargo en la presente investigación se determinó que los y las adolescentes del Ministerio Jóvenes en Victoria, poseen excelentes habilidades sociales, además no existe una relación significativa entre lo anterior dicho y la frecuencia en horas, comprobándose que las habilidades sociales de los jóvenes no van a ser determinadas ni de manera positiva o negativa por las horas que los mismos invierten en conectarse a Facebook. 
Agüero

A partir de los resultados se determinó que no existe una relación directa entre las habilidades sociales percibidas y las horas invertidas en la red social. Si se cumple con la hipótesis planteada de que a mayor tiempo invertido en el uso de la red social Facebook, las y los adolescentes presentan mayor déficit de habilidades sociales.

No obstante, sí se podrían determinar que los jóvenes que entre más agreguen y tengan de amigos, personas que conozcan en la vida real y menos utilicen Facebook para compartir problemas, van a tener más habilidades sociales, además, es importante para los jóvenes, la percepción que pueden tener sus amigos con respecto a lo que publican en Facebook y también la cantidad de amigos que tienen en Facebook.

Las habilidades sociales van a ser influenciadas por muchos factores más, como mencionan las investigadoras Hidalgo y Abarca (1999), "que el comportamiento social constituye un aprendizaje continuo de patrones cada vez más complejos que van a incluir aspectos cognitivos, afectivos, sociales y morales que se van adquiriendo conforme el sujeto va madurando y aprendiendo a interactuar con el medio social" o como menciona Caballo (1995) "la habilidad social es una característica de la conducta, no de la persona, es una característica específica a la persona y a la situación, no universal, deben contemplarse el contexto cultural del individuo para escoger libremente su acción y es una característica de la conducta socialmente eficaz, no dañina". Por lo tanto a la hora de analizar las habilidades sociales de los y las adolescentes se tienen que tomar en cuenta todos los factores anteriores y no sólo determinarse por la cantidad de horas en las que accede a una red social.

Con respecto a las relaciones sociales en Facebook, Jiménez y Pantoja (2007), mencionan que las personas que frecuentan el uso de Facebook son más propensos a contactar personas desconocidas, en cambio los sujetos que no utilizan mucho la red social, 
utilizan estos medios con personas conocidas a ellos, además de utilizarlo también para obtener información y mantener actualizados.

Lo anterior se cumple con los resultados brindados por la presente investigación en donde existe una relación positiva en cuanto a la cantidad de horas que invierten los usuarios y agregar a los amigos de sus amigos y a la gente que no conocen, siendo así que entre más horas estén conectados en Facebook, mas personas desconocidas agregarán, sin embargo esto no necesariamente se podría asociar a una falta de habilidades sociales por parte de los adolescentes, sino simplemente a que debido a las gran cantidad de horas en las que pertenecen conectados pueden interactuar con más personas.

Referente a los usos que los usuarios practican al estar conectados a Facebook, se determinó que la frecuencia del uso de Facebook influye positivamente en la medida en que las personas usen de una manera frecuente las aplicaciones, los usos y los motivos de usarlos. Lo anterior se relaciona con lo propuesto por Benavides y Paladines (2011), en donde expresan que las actividades más frecuentes al conectarse a internet es entrar a las redes sociales en donde lo más común es chatear y estar en contacto u observar lo que publican sus amigos y amigas o familiares.

Sin embargo no hace referencia a la relación que tiene esto con las horas que invierten los y las jóvenes en internet. En esta investigación si se pudo asegurar con certeza que los y las adolescentes que utilizan con frecuencia Facebook son más propensos a utilizar de una manera continua las diferentes utilidades de la misma red social.

\section{Referencias}


Agüero

Barrantes, R. (2000). Investigación: un camino al conocimiento un enfoque cualitativo y cuantitativo. Costa Rica: EUNED.

Benavides, A., Paladines, F. (2011). Uso de otras Formas de Comunicación. Consumo de Medios en los estudiantes universitarios. Análisis de Internet y Teléfono Móvil. Razón y Palabra, 77, 1-36.

Borja, C. (2010). Las redes sociales: lo que hacen sus hijos en internet. España: Club Universitario.

Caballo, V. (1995). Manual de técnicas y modificación de Conducta. ( $3^{\mathrm{a}}$ ed.) Madrid: Siglo XXI Editores.

Caballo, V. (2007). Manual de evaluación y entrenamiento de las Habilidades Sociales. Siglo XXI Editores: Madrid

Castillo, M., Sáenz, L. (2012). Facebook: una posible herramienta de investigación. Rev. Electrónica de estudiantes Esc. de psicología, Univ. de Costa Rica. 7(1): 211-216

Chaves, R. (2005). Relaciones Interpersonales: en el contexto educativo dirigido a los adolescentes del Colegio Mont Berkeley Intl. Proyecto de Graduación para Optar por al Grado de Licenciatura en Psicología. Universidad de Iberoamérica. Facultad de Psicología, San José, Costa Rica.

Dulando, E. (2000). El adolescente. (1 $1^{\mathrm{a}}$ ed.) México: Mc Graw Hill

Gil, S. (2005). Desarrollo de un Programa de Habilidades Sociales para la Prevención de Conductas Sociales Desadaptativas. Proyecto de Graduación para Optar por al Grado de Licenciatura en Psicología. Universidad de Iberoamérica. Facultad de Psicología, San José, Costa Rica.

Hernandez, R. Fernandez, C \& Baptista, P. (2006) Metodología de la Investigación. (4ª ed). México: McGraw Hill 
Herrera, M. et al. (2010). La Adicción a Facebook Relacionada con la Baja Autoestima, la Depresión y la Falta de Habilidades Sociales. Psicología Iberoamericana, 18, 6-18.

Hidalgo, C. \& Abarca, N. (1999). Comunicación interpersonal: programa de entrenamiento en habilidades sociales. ( $3^{\mathrm{a}}$ ed). Colombia: Alfaomega.

Inglés, C., Méndez, F., Hidalgo, M. (2000). Cuestionario de Evaluación de Dificultades Interpersonales en la Adolescencia. Psicothema, 12, 390-398.

Jensen, J. (2008). Adolescencia y adultez emergente: un enfoque cultural. México :Prentice Hall

Jiménez, L. \& Pantoja, A. V. (2007). Autoestima y relaciones Interpersonales en sujetos adictos a Internet. Psicología-Segunda Época, 26(1), 78- 89.

Krauskopf, D. (1998). Adolescencia y Educación. 2a. Ed. Costa Rica: EUNED.

Las Heras, J. (2012). La adicción de los adolescentes a Internet: ¿La incipiente epidemia del siglo 21?. Instituto de Políticas Públicas. 8, 1-7.

Nielsen (2010). Social Networks/Blogs Account for One in Every Four and a Half Minutes Online, de http://blog.Nielsen.com/nielsenwire/online_mobile/social-mediaaccounts-for-22-percent-of-time-online/

Papalia, D. (2001). Psicología del desarrollo. ( $8^{\mathrm{a}}$ ed). Colombia: Mc Graw Hill

Pérez, R., Torres, D., Rumoroso, A. (2010). La auto-valoración de las competencias y el uso mediático en niños y niñas costarricenses. El caso particular de la internet. Global Media Journal México, 7, 24-39.

Ross et al. (2009). Personality and motivations associated with Facebook use. Computers in Human Behavior . 25, 578-586. 
Agüero

Sanchez, C. (2009). Entrenamiento en Habilidades Sociales a estudiantes de licenciatura en

Psicología. Proyecto de Graduación para optar por la Licenciatura en Psiscología. UNIBE.

Sheldon, P. (2008). Student Favorite: Facebook and Motives for its use. Southwestern Mass Communication Journal, 23, 39-54.

Socialbakers. (2012). Costa Rica Facebook Statistics, de http://www.socialbakers.com/facebook-statistics/costa-rica.

UNIMER (2010) 1 de cada 5 ticos usa redes Sociales. Recuperado de: http://www.unimercentroamerica.com/biblioteca_virtual/Redes_Sociales

Young, K. (2009). Online Social Networking: An Australian Perspective. International Journal of EmergingTechnologies \& Society, 7 (1), 39-57.

Zywica, J. \& Danowski, J. (2008). The Faces of Facebookers: Investigating Social Enhancement and Social Compensation Hypotheses; Predicting Facebook and Offline Popularity from Sociability and Self-Esteem, and Mapping the Meanings of Popularity with Semantic Networks. Journal of Computer-Mediated Communication. $14,1-34$ 